\title{
Correction: Systematic Analysis of Head-to-Head Gene Organization: Evolutionary Conservation and Potential Biological Relevance
}

Yuan-Yuan Li, Hui Yu, Zong-Ming Guo, Ting-Qing Guo, Kang Tu, Yi-Xue Li

DOI: 10.1371/journal.pcbi.0020074.

In PLoS Computational Biology, volume 2, issue 7:

The dataset links located in Materials and Methods are not active and should be replaced by the following:

ftp://ftp.ncbi.nih.gov/genomes/H_sapiens/maps/mapview/BUILD.35.1 should be http://rd.plos.org/10.1371_journal.pcbi. 0020074_01

ftp://ftp.ncbi.nih.gov/genomes/M_musculus/mapview/Build should be http://rd.plos.org/10.1371_journal.pcbi.0020074_02

$\mathrm{ftp} / / / \mathrm{ftp}$. ncbi.nih.gov/genomes/R_norvegicus/mapview/Build should be http://rd.plos.org/10.1371_journal.pcbi.0020074_03

ftp://ftp.ensembl.org/pub/current_human/data/fasta/pep should be http://rd.plos.org/10.1371_journal.pcbi.0020074_04

ftp://ftp.ensembl.org/pub/current_chicken/data/fasta/pep should be http://rd.plos.org/10.1371_journal.pcbi.0020074_05

ftp://ftp.ensembl.org/pub/current_chicken/data/mysql/gallus_gallus_core_31_1g should be http://rd.plos.org/10.1371_journal. pcbi.0020074_06

This correction note may be found online at DOI: 10.1371/journal.pcbi.0020112.

Published August 25, 2006.

Citation: (2006) Correction: Systematic analysis of head-to-head gene organization: Evolutionary conservation and potential biological relevance. PLoS Comput Biol 2(8): e112. DOI: 10.1371/journal.pcbi.0020112. 